\title{
On Topological Descriptors of Certain Metal-Organic Frameworks
}

\author{
Peng Xu, ${ }^{1,2}$ Mehran Azeem $\mathbb{D}^{3},{ }^{3}$ Muhammad Mubashir Izhar, ${ }^{4}$ Syed Mazhar Shah, ${ }^{2}$ \\ Muhammad Ahsan Binyamin $\mathbb{D}^{5},{ }^{5}$ and Adnan Aslam $\mathbb{D}^{3}$ \\ ${ }^{1}$ Institute of Computational Science and Technology, Guangzhou University, Guangzhou, China \\ ${ }^{2}$ School of Computer Science of Information Technology, Qiannan Normal University for Nationalities, Duyun 558000, China \\ ${ }^{3}$ University of Engineering and Technology (RCET), Lahore, Pakistan \\ ${ }^{4}$ Department of Mathematics, Minhaj University Lahore, Lahore, Pakistan \\ ${ }^{5}$ Department of Mathematics, GC University Faisalabad, Faisalabad, Pakistan
}

Correspondence should be addressed to Muhammad Ahsan Binyamin; ahsanbanyamin@gmail.com

Received 24 September 2020; Revised 20 October 2020; Accepted 28 October 2020; Published 16 November 2020

Academic Editor: Cláudia G. Silva

Copyright () 2020 Peng Xu et al. This is an open access article distributed under the Creative Commons Attribution License, which permits unrestricted use, distribution, and reproduction in any medium, provided the original work is properly cited.

Topological indices are numerical numbers that represent the topology of a molecule and are calculated from the graphical depiction of the molecule. The importance of topological indices is due to their use as descriptors in QSPR/QSAR modeling. QSPRs (quantitative structure-property relationships) and QSARs (quantitative structure-activity relationships) are mathematical correlations between a specified molecular property or biological activity and one or more physicochemical and/or molecular structural properties. In this paper, we give explicit expressions of some degree-based topological indices of two classes of metalorganic frameworks (MOFs), namely, butylated hydroxytoluene- (BHT-) based metal-organic $(\mathrm{M}=\mathrm{Co}, \mathrm{Fe}, \mathrm{Mn}, \mathrm{Cr})(\mathrm{MBHT})$ frameworks and $\mathrm{M}_{1} \mathrm{TPy} P-\mathrm{M}_{2}\left(\mathrm{TPyP}=5,10,15,20\right.$-tetrakis(4-pyridyl)porphyrin and $\mathrm{M}_{1}, \mathrm{M}_{2}=\mathrm{Fe}$ and Co) MOFs.

\section{Introduction}

Metal-organic frameworks (MOFs) are defined by their regular array of metal cores and organic linkers in a threedimensional framework. In MOFs, all metal cores are connected to organic linkers to construct networks which can contain a range of guest molecules. The first MOF was reported in 1959 by Kinoshita et al. [1]. MOFs receive attention due to the use of reticular chemistry for their design and synthesis [2]. After that, thousands of MOFs have been synthesized and broaden the scope of their potential applications. MOFs have shown applications in the area of gas catalysis [3-5], delivery of drugs [6-8], sensing [9, 10], separation [11, 12], storage [13-15], and adsorption [16-20]. There are many open sites in MOFs that are capable of capturing industrial flue gases, such as $\mathrm{CO}_{2}, \mathrm{SO}_{2}, \mathrm{NO}, \mathrm{CO}$, and $\mathrm{NO}_{2}$ [21-23]. These flue gases have the harmful effect on the environment. For instance, the emission of $\mathrm{CO}_{2}$ from the burning of fossil fuel is the major issue for climate change and greenhouse effect [24]. $\mathrm{SO}_{2}$ and $\mathrm{NO}_{2}$ are the main reason for the induction of acid rain as well as smog [25], and $\mathrm{CO}$ and NO are asphyxiants for humans [26]. Therefore, there is a need to develop an efficient strategy to limit the release of these hazardous gases to improve the worsening environmental quality. MOFs have shown a great potential to trap $\mathrm{CO}_{2}$ in their porous structure. MOF-74 has the ability to capture $\mathrm{CO}_{2}$ at room temperature and low pressure. For more details on the ability of MOFs to capture flue gas molecules, refer [27-29].

In mathematical chemistry and in chemical graph theory, the structural formula of a chemical compound is represented by a molecular graph where the vertices are represented by the atoms, and the edges are represented by the bonds between the vertices. Let $G(V(G), E(G))$ be a molecular graph, where $V(G)$ and $E(G)$ denote the vertex set and edge set, respectively. Two vertices $x$ and $y$ are adjacent if they are end vertices of a common edge $e=x y$. The set of neighbors of a vertex $x$ is denoted by $N_{x}$ and is defined as $N_{x}=\{y \in V(G): x y \in E(G)\}$. The degree of a vertex $x$ is symbolized by $d_{x}$ and is the cardinality of the set 
$N_{x}$. Let $S_{x}$ denote the sum of degrees of the neighbor of the vertex $x$, that is, $S_{x}=\left\{\sum d_{y}: y \in N_{x}\right\}$. For undefined terminologies related to graph theory, we refer the reader to [30].

Molecular descriptors play an important role in the quantitative description of the molecular structure in finding appropriate predictive models. Molecular descriptors are terms that characterize a particular aspect of a molecule [31] and can be categorized into global and local according to the way the molecular structure is characterized. The topological indices (TIs) are among the most useful molecular descriptors known today [32-34]. These descriptors are numerical values related to chemical structure to correlate chemical structure with different physical properties, chemical reactivity, or biological activity [31]. First, topological index was introduced by Weiner [35], named Weiner index, and is defined as the number of carbon-carbon between all pairs of carbon atoms in an alkane. After 25 years, the introduction of connectivity indices and their application motivated the study of such descriptors [31]. Most of the known topological indices are global molecular descriptors. It means these indices characterize the molecule as a whole. For example, it can describe its branching or shape of the entire structure. The first degree-based topological index was introduced by Randic [36] in 1975. It is denoted by $R_{-1 / 2}(G)$ and is defined as

$$
R_{-1 / 2}(G)=\sum_{x y \in E(G)} \frac{1}{\sqrt{d_{x} d_{y}}} .
$$

Randic observed that there is a very good correlation between Randic index and certain physical/chemical properties of alkanes: boiling points, enthalpies of formation, chromatographic retention times, surface areas, and parameters in the Antoine equation for vapor pressure. Later, in 1998, Bollobás and Erd $\square$ s [37] generalized this index by replacing $-1 / 2$ with any real number $\alpha$, which is called the general Randic index:

$$
R_{\alpha}(G)=\sum_{x y \in E(G)}\left(d_{x} d_{y}\right)^{\alpha} .
$$

The first and second Zagreb indices were introduced by Gutman et al. [38] and were applied to the branching problem in 1972. The first and second Zagreb indices are denoted and defined as

$$
\begin{aligned}
& M_{1}(G)=\sum_{x y \in E(G)}\left(d_{x}+d_{y}\right), \\
& M_{2}(G)=\sum_{x y \in E(G)}\left(d_{x} \times d_{y}\right) .
\end{aligned}
$$

Recently, Shirdel et al. [39] proposed the hyper-Zagreb index:

$$
\operatorname{HM}(G)=\sum_{x y \in E(G)}\left[d_{x}+d_{y}\right]^{2} .
$$

The Zagreb indices and their variants have been used to study molecular complexity [40-44], chirality [45], ZE- isomerism [46], and heterosystems [47] whilst the overall Zagreb indices exhibit potential applicability for deriving multilinear regression models. Various researchers also use the Zagreb indices in their QSPR and QSAR studies [48-53].

In 2009, Zhou and Trinajstic [54] introduced the sum connectivity index. It was observed that the sum connectivity index correlates well with the $\pi$ electron energy of hydrocarbons. It is denoted and defined as

$$
\operatorname{SCI}(G)=\sum_{x y \in E(G)} \frac{1}{\sqrt{d_{x}+d_{y}}} .
$$

Recently, Zhou and Trinajstic [55] extended this concept to the general sum connectivity index. The general sum connectivity index is defined as

$$
\chi_{\alpha}(G)=\sum_{x y \in E(G)}\left(d_{x}+d_{y}\right)^{\alpha} .
$$

Atom-bond connectivity (ABC) index was introduced by Estrada et al. [56] in 1998 which is denoted as

$$
\operatorname{ABC}(G)=\sum_{x y \in E(G)} \sqrt{\frac{d_{x}+d_{y}-2}{d_{x} d_{y}}} .
$$

The ABC index provides a good model for the stability of linear and branched alkanes as well as the strain energy of cycloalkanes $[56,57]$.

Recently, the well-known connectivity topological index is geometric-arithmetic (GA)index which was introduced by Vukičević and Furtula in [58]. For a graph $G$, the GA index is denoted and defined as

$$
\mathrm{GA}(G)=\sum_{x y \in E(G)} \frac{2 \sqrt{d_{x} d_{y}}}{d_{x}+d_{y}} .
$$

It has been demonstrated on the example of octane isomers that GA index is well correlated with a variety of physicochemical properties.

The fourth version of the atom-bond connectivity index $\left(\mathrm{ABC}_{4}\right)$ was introduced by Ghorbani and Hosseinzadeh [45] in 2010 and is defined as

$$
\mathrm{ABC}_{4}(G)=\sum_{x y \in E(G)} \sqrt{\frac{S_{x}+S_{y}-2}{S_{x} S_{y}}} .
$$

The fifth version of the topological index GA is proposed by Graovac et al. [59] in 2011 which is expressed as

$$
\mathrm{GA}_{5}(G)=\sum_{x y \in E(G)} \frac{2 \sqrt{S_{x} S_{y}}}{S_{x}+S_{y}} .
$$

For more details on the computation of topological indices, we refer the readers to [60-65].

The main aim of this work is to compute the degreebased topological indices of two classes of MOFs. The computed topological indices can be used in QSPR/QSAR studies to improve the physical/chemical properties of the considered MOFs. The same technique can be used to 
compute the degree-based topological indices of other classes of MOFs. In the next section, we compute the abovedefined topological indices of $\mathrm{M}_{1} \mathrm{TPyP}-\mathrm{M}_{2}$ metal-organic frameworks.

\section{Topological Aspects of $2 D$ Structure of $M_{1}$ TPyP $-M_{2}$ Metal-Organic Frameworks}

Wurster et al. [66] prepared $\mathrm{M}_{1} \mathrm{TPyP}-\mathrm{M}_{2} \mathrm{MOF}$ and observed that these MOFs have two metal centers at two distinguished coordination environments. They are bimetallic MOFs. Depending on $\mathrm{M}_{1}$ and $\mathrm{M}_{2}$, these MOFs become either hetero- or homobimetallic. The performance of these MOFs was observed in oxygen evolution reactions, which generate $\mathrm{O}_{2}$ from water. They reported that the catalytic activity of metalloporphyrins was lower than that of heterobimetallic MOFs. The 2D structure of $\mathrm{M}_{1} \mathrm{TPyP}-\mathrm{M}_{2}$ MOFs is depicted in Figure 1. We denote the graph of $\mathrm{M}_{1}$ TPyP $-\mathrm{M}_{2}$ MOFs by $\mathrm{G}_{1}(c, d)$, where $c$ and $d$ represent the number of unit cell in each row and column, respectively. The $2 \mathrm{D}$ structure of the molecular graph of $\mathscr{G}_{1}(2,2)$ is shown in Figure 1.

A simple calculation shows that $\mathscr{G}_{1}(c, d)$ has $74 c d$ vertices and $88 c d-2 c-2 d+1$ edges. First, the general
Randic connectivity and general sum connectivity indices of $\mathscr{G}_{1}(c, d)$ were computed.

Theorem 1. The Randic connectivity index and general sum connectivity indices of the graph $\mathscr{G}_{1}(c, d)$ are

$$
\begin{aligned}
R_{\alpha}\left(\mathscr{G}_{1}(c, d)\right)= & (24 c d+1)(3)^{\alpha}+(6 c+6 d-6)(6)^{\alpha} \\
& +(56 c d-4 c-4 d+2)(9)^{\alpha} \\
& +(8 c d-4 c-4 d+4)(12)^{\alpha}, \\
\chi_{\alpha}\left(\mathscr{G}_{1}(c, d)\right)= & (24 c d+1)(4)^{\alpha}+(6 c+6 d-6)(5)^{\alpha} \\
& +(56 c d-4 c-4 d+2)(6)^{\alpha} \\
& +(8 c d-4 c-4 d+4)(7)^{\alpha} .
\end{aligned}
$$

Proof. To compute the general Randic connectivity index and general sum connectivity index, we need to find the edge partition of $\mathscr{G}_{1}(c, d)$ depending on the degree of end vertices. This partition is given in Table 1 . Now using the values of the edge partition in the definition of these indices, we get the result as follows:

$$
\begin{aligned}
R_{\alpha}\left(\mathscr{G}_{1}(c, d)\right)= & \sum_{x y \in \mathscr{E}(1,3)\left(\mathscr{G}_{1}(c, d)\right)}\left(d_{x} d_{y}\right)^{\alpha}+\sum_{x y \in \mathscr{E}_{(2,3)}\left(\mathscr{G}_{1}(c, d)\right)}\left(d_{x} d_{y}\right)^{\alpha}+\sum_{x y \in \mathscr{C}_{(3,3)}\left(\mathscr{G}_{1}(c, d)\right)}\left(d_{x} d_{y}\right)^{\alpha} \\
& +\sum_{x y \in \mathscr{E}_{(3,4)}\left(\mathscr{G}_{1}(c, d)\right)}\left(d_{x} d_{y}\right)^{\alpha}=(24 c d+1)(3)^{\alpha}+(6 c+6 d-6)(6)^{\alpha}+(56 c d-4 c-4 d+2)(9)^{\alpha} \\
& +(8 c d-4 c-4 d+4)(12)^{\alpha}, \\
\chi_{\alpha}\left(\mathscr{G}_{1}(c, d)\right)= & \sum_{x y \in \mathscr{E}_{(1,3)}\left(\mathscr{G}_{1}(c, d)\right)}\left(d_{x}+d_{y}\right)^{\alpha}+\sum_{x y \in \mathscr{E}_{(2,3)}\left(\mathscr{G}_{1}(c, d)\right)}\left(d_{x}+d_{y}\right)^{\alpha}+\sum_{x y \in \mathscr{E}_{(3,3)}\left(\mathscr{G}_{1}(c, d)\right)}\left(d_{x}+d_{y}\right)^{\alpha} \\
& +\sum_{x y \in \mathscr{C}_{(3,4)}\left(\mathscr{G}_{1}(c, d)\right)}\left(d_{x}+d_{y}\right)^{\alpha}=(24 c d+1)(4)^{\alpha}+(6 c+6 d-6)(5)^{\alpha}+(56 c d-4 c-4 d+2)(6)^{\alpha} \\
& +(8 c d-4 c-4 d+4)(7)^{\alpha} .
\end{aligned}
$$

Corollary 1. The values of Randic connectivity, first and second Zagreb, hyper-Zagreb, and sum connectivity indices can be computed from the above theorem by using the value of $\alpha=-1 / 2,-1,1,2$.

$$
\begin{aligned}
& R_{-1 / 2}\left(\mathscr{G}_{1}(c, d)\right)=\left(\frac{28 \sqrt{3}}{3}+\frac{56}{3}\right) c d+\left(\sqrt{6}-\frac{2 \sqrt{3}}{3}-\frac{4}{3}\right) c+\left(\sqrt{6}-\frac{2 \sqrt{3}}{3}-\frac{4}{3}\right) d+\sqrt{3}-\sqrt{6}+\frac{2}{3} \\
& M_{1}\left(\mathscr{G}_{1}(c, d)\right)=488 c d-22 c-22 d+14 \\
& M_{2}\left(\mathscr{G}_{1}(c, d)\right)=672 c d-48 c-48 d+33 \\
& \operatorname{SCI}\left(\mathscr{G}_{1}(c, d)\right)=\left(\frac{28 \sqrt{6}}{3}+\frac{8 \sqrt{7}}{7}\right) c d+\left(\frac{\sqrt{6} 5}{5}-\frac{2 \sqrt{6}}{3}-\frac{4 \sqrt{7}}{7}\right) c+\left(\frac{\sqrt{6} 5}{5}-\frac{2 \sqrt{6}}{3}-\frac{4 \sqrt{7}}{7}\right) d+\frac{\sqrt{6}}{3}-\frac{6 \sqrt{5}}{5}+\frac{4 \sqrt{7}}{7}+\frac{1}{2} \\
& \operatorname{HM}\left(\mathscr{G}_{1}(c, d)\right)=2792 c d-190 c-190 d+134
\end{aligned}
$$




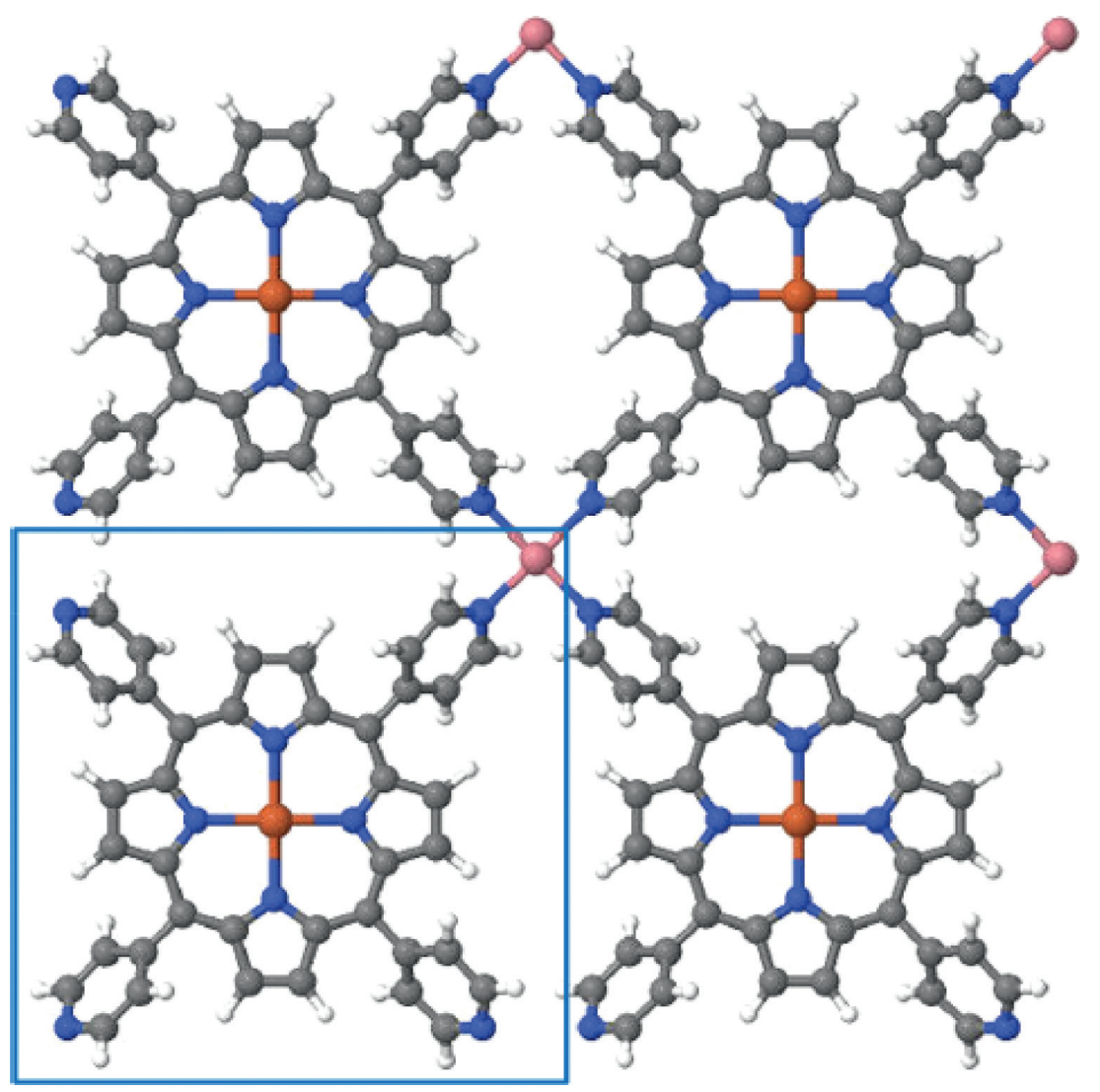

Figure 1: $(2 \times 2)$ supercell of $\mathrm{M}_{1} \mathrm{TPy}-\mathrm{M}_{2} \mathrm{MOFs}\left(\mathrm{M}_{1}\right.$ and $\mathrm{M}_{2}=\mathrm{Fe}$ and Co $)$.

TABLE 1: The partition of edge set of graph $\mathscr{G}_{1}(c, d)$ based on degrees of end vertices of each edge.

\begin{tabular}{lr}
\hline$\left(d_{x}, d_{y}\right)$ where $x y \in E\left(\mathscr{G}_{1}(c, d)\right)$ & Number of edges \\
\hline$(1,3)$ & $24 c d+1$ \\
$(2,3)$ & $6 c+6 d-6$ \\
$(3,3)$ & $56 c d-4 c-4 d+2$ \\
$(3,4)$ & $8 c d-4 c-4 d+4$ \\
\hline
\end{tabular}

In the next theorem, the $\mathrm{ABC}$ and $\mathrm{GA}$ indices of $\mathscr{G}_{1}(c, d)$ were computed.
Theorem 2. The $A B C$ and $G A$ indices of the graph $\mathscr{G}_{1}(c, d)$ are

$$
\begin{aligned}
& \mathrm{ABC}\left(\mathscr{G}_{1}(c, d)\right)=\left(8 \sqrt{6}+\frac{4 \sqrt{15}}{3}+\frac{112}{3}\right)+c d+\left(3 \sqrt{2}-\frac{2 \sqrt{15}}{3}-\frac{8}{3}\right) c+\left(3 \sqrt{2}-\frac{2 \sqrt{15}}{3}-\frac{8}{3}\right) d+\frac{\sqrt{6}}{3}+\frac{2 \sqrt{15}}{3}-3 \sqrt{2}+\frac{4}{3} \\
& \mathrm{GA}\left(\mathscr{G}_{1}(c, d)\right)=\left(\frac{116 \sqrt{3}}{7}+56\right) c d+\left(\frac{12 \sqrt{6}}{5}-\frac{16 \sqrt{3}}{7}-4\right) c+\left(\frac{12 \sqrt{6}}{5}-\frac{16 \sqrt{3}}{7}-4\right) d+\frac{39 \sqrt{3}}{14}-\frac{12 \sqrt{6}}{5}+2 .
\end{aligned}
$$


Proof. By using the values of the edge partition given in Table 1 in the definition of the ABC index, this index can be determined as follows:

$$
\begin{aligned}
\operatorname{ABC}\left(\mathscr{G}_{1}(c, d)\right) & =(24 c d+1)\left(\frac{\sqrt{6}}{3}\right)+(6 c+6 d-6)\left(\frac{\sqrt{2}}{2}\right)+(56 c d-4 c-4 d+2)\left(\frac{2}{3}\right)+(8 c d-4 c-4 d+4)\left(\frac{\sqrt{15}}{6}\right) \\
& =\left(8 \sqrt{6}+\frac{4 \sqrt{15}}{3}+\frac{112}{3}\right) c d+\left(3 \sqrt{2}-\frac{2 \sqrt{15}}{3}-\frac{8}{3}\right) c+\left(3 \sqrt{2}-\frac{2 \sqrt{15}}{3}-\frac{8}{3}\right) d+\frac{\sqrt{6}}{3}+\frac{2 \sqrt{15}}{3}-3 \sqrt{2}+\frac{4}{3}
\end{aligned}
$$

Similarly, the GA index can be calculated as

$$
\begin{aligned}
\mathrm{GA}\left(\mathscr{G}_{1}(c, d)\right) & =(24 c d+1)\left(\frac{\sqrt{3}}{2}\right)+(6 c+6 d-6)\left(\frac{2 \sqrt{6}}{5}\right)+(56 c d-4 c-4 d+2)(1)+(8 c d-4 c-4 d+4)\left(\frac{4 \sqrt{3}}{7}\right) \\
& =\left(\frac{116 \sqrt{3}}{7}+56\right) c d+\left(\frac{12 \sqrt{6}}{5}-\frac{16 \sqrt{3}}{7}-4\right) c+\left(\frac{12 \sqrt{6}}{5}-\frac{16 \sqrt{3}}{7}-4\right) d+\frac{39 \sqrt{3}}{14}-\frac{12 \sqrt{6}}{5}+2 .
\end{aligned}
$$

Finally, the expression of $\mathrm{ABC}_{4}$ and $\mathrm{GA}_{5}$ indices are calculated in the next theorem.

$$
\begin{aligned}
\mathrm{ABC}_{4}\left(\mathscr{G}_{1}(c, d)\right)= & \left(\frac{4 \sqrt{42}}{7}+\frac{16 \sqrt{42}}{7}+\frac{4 \sqrt{170}}{15}+\frac{16 \sqrt{2}}{3}+\frac{24 \sqrt{3}}{7}+\frac{4 \sqrt{6}}{3}+\frac{16}{3}\right) c d \\
& +\left(\frac{2 \sqrt{10}}{3}+\frac{2 \sqrt{14}}{3}-\frac{4 \sqrt{42}}{7}-\frac{8 \sqrt{42}}{7}+\frac{\sqrt{182}}{7}+\frac{2 \sqrt{462}}{21}-\frac{8 \sqrt{3}}{7}-\frac{2 \sqrt{6}}{3}+1\right) c \\
& +\left(\frac{2 \sqrt{10}}{3}+\frac{2 \sqrt{14}}{3}-\frac{4 \sqrt{42}}{7}-\frac{8 \sqrt{42}}{7}+\frac{\sqrt{182}}{7}+\frac{2 \sqrt{462}}{21}-\frac{8 \sqrt{3}}{7}-\frac{2 \sqrt{6}}{3}+1\right) d \\
& +\frac{4 \sqrt{42}}{7}-\frac{\sqrt{14}}{3}-\frac{\sqrt{10}}{3}+\frac{2 \sqrt{42}}{7}-\frac{2 \sqrt{182}}{7}-\frac{\sqrt{462}}{21}+\frac{8 \sqrt{3}}{7}+\frac{2 \sqrt{6}}{3}-2, \\
\mathrm{GA}_{5}\left(\mathscr{G}_{1}(c, d)\right)= & \left(6 \sqrt{7}+\frac{48 \sqrt{10}}{19}+\frac{24 \sqrt{21}}{5}+\frac{16 \sqrt{30}}{11}+\frac{16 \sqrt{70}}{17}+24\right) c d \\
& +\left(\frac{8 \sqrt{2}}{3}+\frac{8 \sqrt{3}}{7}+\frac{16 \sqrt{14}}{15}-\frac{12 \sqrt{21}}{5}-\frac{8 \sqrt{30}}{11}+\frac{8 \sqrt{42}}{13}-\frac{16 \sqrt{70}}{17}\right) c \\
& +\left(\frac{8 \sqrt{2}}{3}+\frac{8 \sqrt{3}}{7}+\frac{16 \sqrt{14}}{15}-\frac{12 \sqrt{21}}{5}-\frac{8 \sqrt{30}}{11}+\frac{8 \sqrt{42}}{13}-\frac{16 \sqrt{70}}{17}\right) d \\
& +\frac{3 \sqrt{21}}{5}-\frac{16 \sqrt{3}}{7}-\frac{32 \sqrt{14}}{15}-\frac{4 \sqrt{2}}{3}+\frac{8 \sqrt{30}}{11}-\frac{4 \sqrt{42}}{13}+\frac{16 \sqrt{70}}{17}+2 .
\end{aligned}
$$

Proof. To compute the values of $\mathrm{ABC}_{4}$ and $\mathrm{GA}_{5}$ indices, we need to find the partition of the edge set based on the sum of degrees of the neighbors of the end vertices of each edge.
This partition is presented in Table 2. Now, using the values in the definition of $\mathrm{ABC}_{4}$ index, this index can be calculated as 


$$
\begin{aligned}
\mathrm{ABC}_{4}\left(\mathscr{G}_{1}(c, d)\right)= & (4 c+4 d-2)\left(\frac{\sqrt{14}}{6}\right)+(24 c d-12 c-12 d+3)\left(\frac{2 \sqrt{42}}{21}\right)+(4 c+4 d-2)\left(\frac{\sqrt{10}}{6}\right) \\
& +(4 c+4 d-2)\left(\frac{\sqrt{426}}{42}\right)+(2 c+2 d-4)\left(\frac{1}{2}\right)+(12 c d-4 c-4 d+4)\left(\frac{2 \sqrt{3}}{7}\right)+(4 c+4 d-8)\left(\frac{\sqrt{182}}{28}\right) \\
& +(16 c d)\left(\frac{\sqrt{2}}{3}\right)+(8 c d-8 c-8 d+8)\left(\frac{\sqrt{42}}{14}\right)+(12 c d)\left(\frac{4}{9}\right)+(8 c d)\left(\frac{\sqrt{170}}{30}\right) \\
& +(8 c d-4 c-4 d+4)\left(\frac{\sqrt{6}}{6}\right)=\left(\frac{4 \sqrt{42}}{7}+\frac{16 \sqrt{42}}{7}+\frac{4 \sqrt{170}}{15}+\frac{16 \sqrt{2}}{3}+\frac{24 \sqrt{3}}{7}+\frac{4 \sqrt{6}}{3}+\frac{16}{3}\right) c d \\
& +\left(\frac{2 \sqrt{10}}{3}+\frac{2 \sqrt{14}}{3}-\frac{4 \sqrt{42}}{7}-\frac{8 \sqrt{42}}{7}+\frac{\sqrt{182}}{7}+\frac{2 \sqrt{462}}{21}-\frac{8 \sqrt{3}}{7}-\frac{2 \sqrt{6}}{3}+1\right) c \\
& +\left(\frac{2 \sqrt{10}}{3}+\frac{2 \sqrt{14}}{3}-\frac{4 \sqrt{42}}{7}-\frac{8 \sqrt{42}}{7}+\frac{\sqrt{182}}{7}+\frac{2 \sqrt{462}}{21}-\frac{8 \sqrt{3}}{7}-\frac{2 \sqrt{6}}{3}+1\right) d \\
& +\frac{4 \sqrt{42}}{7}-\frac{\sqrt{14}}{3}-\frac{\sqrt{10}}{3}+\frac{2 \sqrt{42}}{7}-\frac{2 \sqrt{182}}{7}-\frac{\sqrt{462}}{21}+\frac{8 \sqrt{3}}{7}+\frac{2 \sqrt{6}}{3}-2 .
\end{aligned}
$$

Similarly, the value of $\mathrm{GA}_{5}$ index can be calculated as

$$
\begin{aligned}
\mathrm{GA}_{5}\left(\mathscr{G}_{1}(c, d)\right)= & (4 c+4 d-2)\left(\frac{2 \sqrt{2}}{3}\right)+(24 c d-12 c-12 d+3)\left(\frac{\sqrt{21}}{5}\right)+(4 c+4 d-2)(1)+(4 c+4 d-2)\left(\frac{2 \sqrt{42}}{13}\right) \\
& +(2 c+2 d-4)\left(\frac{4 \sqrt{3}}{7}\right)+(12 c d-4 c-4 d+4)(1)+(4 c+4 d-8)\left(\frac{4 \sqrt{14}}{15}\right) \\
& +(16 c d)\left(\frac{3 \sqrt{7}}{8}\right)+(8 c d-8 c-8 d+8)\left(\frac{2 \sqrt{70}}{17}\right)+(12 c d)(1)+(8 c d)\left(\frac{6 \sqrt{10}}{19}\right) \\
& +(8 c d-4 c-4 d+4)\left(\frac{2 \sqrt{30}}{11}\right)=\left(6 \sqrt{7}+\frac{48 \sqrt{10}}{19}+\frac{24 \sqrt{21}}{5}+\frac{16 \sqrt{30}}{11}+\frac{16 \sqrt{70}}{17}+24\right) c d \\
& +\left(\frac{8 \sqrt{2}}{3}+\frac{8 \sqrt{3}}{7}+\frac{16 \sqrt{14}}{15}-\frac{12 \sqrt{21}}{5}-\frac{8 \sqrt{30}}{11}+\frac{8 \sqrt{42}}{13}-\frac{16 \sqrt{70}}{17}\right) c \\
& +\left(\frac{8 \sqrt{2}}{3}+\frac{8 \sqrt{3}}{7}+\frac{16 \sqrt{14}}{15}-\frac{12 \sqrt{21}}{5}-\frac{8 \sqrt{30}}{11}+\frac{8 \sqrt{42}}{13}-\frac{16 \sqrt{70}}{17}\right) d \\
& +\frac{3 \sqrt{21}}{5}-\frac{16 \sqrt{3}}{7}-\frac{32 \sqrt{14}}{15}-\frac{4 \sqrt{2}}{3}+\frac{8 \sqrt{30}}{11}-\frac{4 \sqrt{42}}{13}+\frac{16 \sqrt{70}}{17}+2 . \\
& +(17
\end{aligned}
$$

\section{Topological Aspects of $2 D$ CoBHT (CO) Lattice}

Clough et al. [67] synthesize the $2 \mathrm{D}$ cobalt bis(dithioline) (CoBHT) metal-organic surface. Chakravarty et al. [10] investigated the electronic and magnetic properties of a $2 \mathrm{D}$ metal-organic (MBHT) framework. They observed that all these frameworks are planar, perfect Kagome lattices with a six-fold symmetry. There is a possibility that these MOFs can be used as gas sensors such as $\mathrm{CO}$ sensing. The optimized structure of these MOFs is shown in Figure 2.
We denote the molecular graph of $2 \mathrm{D}$ metalorganic superlattice by $\mathscr{G}_{2}(a, b)$ with $b$ unit cells in each row and $a$ unit cell in each column. Figure 2 depicts the molecular graph $\mathscr{G}_{2}(2,2)$. A simple calculation shows that $\mathscr{G}_{2}(a, b)$ has $27 a b$ vertices and $36 a b-2 a-2 b$ edges. First, we compute the general Randic connectivity and general sum connectivity indices of $\mathscr{G}_{2}(a, b)$.

Theorem 4. The Randic connectivity index and general sum connectivity indices of graph $\mathscr{G}_{2}(a, b)$ are 
TABLE 2: The partition of edge set of graph $\mathscr{G}_{1}(c, d)$ based on sum of degrees of neighbor vertices of end vertices of each edge.

\begin{tabular}{lr}
\hline$\left(S_{x}, S_{y}\right)$ where $x y \in E\left(\mathscr{G}_{1}(c, d)\right)$ & Number of edges \\
\hline$(3,6)$ & $4 c+4 d-2$ \\
$(3,7)$ & $24 c d-12 c-12 d+3$ \\
$(6,6)$ & $4 c+4 d-2$ \\
$(6,7)$ & $4 c+4 d-2$ \\
$(6,8)$ & $2 c+2 d-4$ \\
$(7,7)$ & $12 c d-4 c-4 d+4$ \\
$(7,8)$ & $4 c+4 d-8$ \\
$(7,9)$ & $16 c d$ \\
$(7,10)$ & $8 c d-8 c-8 d+8$ \\
$(9,9)$ & $12 c d$ \\
$(9,10)$ & $8 c d$ \\
$(10,12)$ & $8 c d-4 c-4 d+4$ \\
\hline
\end{tabular}

$$
\begin{aligned}
& R_{\alpha}\left(\mathscr{G}_{2}(a, b)\right)=(2 a+2 b)(3)^{\alpha}+(2 a+2 b)(4)^{\alpha}+(12 a b-2 a-2 b)(6)^{\alpha}+(12 a b-2 a-2 b)(8)^{\alpha}+(12 a b)(9)^{\alpha} \\
& \chi_{\alpha}\left(\mathscr{G}_{2}(a, b)\right)=(2 a+2 b)(4)^{\alpha}+(2 a+2 b)(4)^{\alpha}+(12 a b-2 a-2 b)(5)^{\alpha}+(12 a b-2 a-2 b)(6)^{\alpha}+(12 a b)(6)^{\alpha} .
\end{aligned}
$$

Proof. To compute the general Randic connectivity index and general sum connectivity index, we need to find the edge partition of $\mathscr{G}_{2}(a, b)$ depending on the degree of end vertices. This partition is given in Table 3. Now using the values of the edge partition in the definition of these indices, we get the result as follows:

$$
\begin{aligned}
R_{\alpha}\left(\mathscr{G}_{2}(a, b)\right)= & \sum_{x y \in \mathscr{E}_{(1,3)}\left(\mathscr{G}_{2}(a, b)\right)}\left(d_{x} d_{y}\right)^{\alpha}+\sum_{x y \in \mathscr{E}_{(2,2)}\left(\mathscr{G}_{2}(a, b)\right)}\left(d_{x} d_{y}\right)^{\alpha}+\sum_{x y \in \mathscr{E}_{(2,3)}\left(\mathscr{G}_{2}(a, b)\right)}\left(d_{x} d_{y}\right)^{\alpha}+\sum_{x y \in \mathscr{E}_{(2,4)}\left(\mathscr{G}_{2}(a, b)\right)}\left(d_{x} d_{y}\right)^{\alpha} \\
& +\sum_{x y \in \mathscr{E}_{(3,3)}\left(\mathscr{G}_{2}(a, b)\right)}\left(d_{x} d_{y}\right)^{\alpha} \\
= & (2 a+2 b)(1 \times 3)^{\alpha}+(2 a+2 b)(2 \times 2)^{\alpha}+(12 a b-2 a-2 b)(2 \times 3)^{\alpha}+(12 a b-2 a-2 b)(2 \times 4)^{\alpha} \\
& +(12 a b)(3 \times 3)^{\alpha}=(2 a+2 b)(3)^{\alpha}+(2 a+2 b)(4)^{\alpha}+(12 a b-2 a-2 b)(6)^{\alpha}+(12 a b-2 a-2 b)(8)^{\alpha} \\
& +(12 a b)(9)^{\alpha}, \\
\chi_{\alpha}\left(\mathscr{G}_{2}(a, b)\right)= & \sum \sum^{\alpha}\left(d_{x}+d_{y}\right)^{\alpha}+\sum_{x y \in \mathscr{E}_{(2,3)}\left(\mathscr{G}_{2}(a, b)\right)}\left(\mathscr{G}_{2}(a, b)\right) \\
& +\sum_{x y \in \mathscr{E}_{(2,4)}\left(\mathscr{G}_{2}(a, b)\right)}\left(d_{x}+d_{y}\right)^{\alpha}+\sum_{x y \in \mathscr{E}_{(2,3)}\left(\mathscr{G}_{2}(a, b)\right)}\left(d_{x}+d_{y}\right)^{\alpha} \\
= & (2 a+2 b)(1+3)^{\alpha}+(2 a+2 b)(2+2)^{\alpha}+(12 a b-2 a-2 b)(2+3)^{\alpha}+(12 a b-2 a-2 b)(2+4)^{\alpha} \\
& +(12 a b)(3+3)^{\alpha} .=(2 a+2 b)(4)^{\alpha}+(2 a+2 b)(4)^{\alpha}+(12 a b-2 a-2 b)(5)^{\alpha}+(12 a b-2 a-2 b)(6)^{\alpha}+(12 a b)(6)^{\alpha} .
\end{aligned}
$$

Corollary 2. The values of Randic connectivity, first and second Zagreb, hyper-Zagreb, and sum connectivity indices can be computed from the above theorem by using the value of $\alpha=-1 / 2,-1,1,2$. 


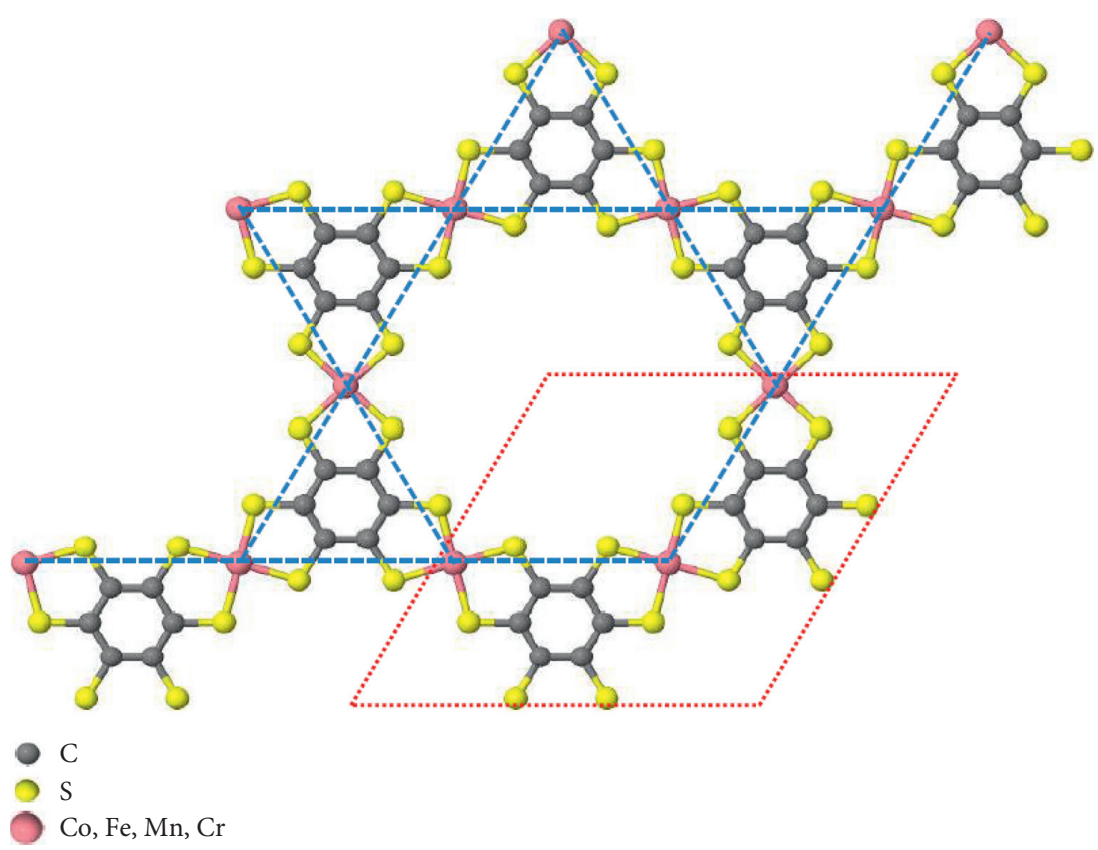

Figure 2: $(2 \times 2)$ supercell of planar MBHT framework.

$$
\begin{aligned}
R_{-1 / 2}\left(\mathscr{G}_{2}(a, b)\right) & =(3 \sqrt{2}+2 \sqrt{6}+4) a b+\left(\frac{2 \sqrt{3}}{3}-\frac{\sqrt{2}}{2}-\frac{6}{3}+1\right) a+\left(\frac{2 \sqrt{3}}{3}-\frac{\sqrt{2}}{2}-\frac{6}{3}+1\right) b \\
M_{1}\left(\mathscr{G}_{2}(a, b)\right) & =204 a b-6 a-6 b \\
M_{2}\left(\mathscr{G}_{2}(a, b)\right) & =276 a b-14 a-14 b \\
\operatorname{SCI}\left(\mathscr{G}_{2}(a, b)\right) & =\left(\frac{12 \sqrt{5}}{5}+4 \sqrt{6}\right) a b+\left(2-\frac{\sqrt{6}}{3}-\frac{2 \sqrt{5}}{5}\right) a+\left(2-\frac{\sqrt{6}}{3}-\frac{2 \sqrt{5}}{5}\right) b \\
\operatorname{HM}\left(\mathscr{G}_{2}(a, b)\right) & =1164 a b-58 a-58 b .
\end{aligned}
$$

In the next theorem, the $\mathrm{ABC}$ and GA indices of $\mathscr{G}_{2}(a, b)$ were computed.

Theorem 5. The $A B C$ and $G A$ indices of the graph $\mathscr{G}_{2}(a, b)$ are

$$
\begin{aligned}
\operatorname{ABC}\left(\mathscr{G}_{2}(a, b)\right)= & \left(\frac{12 \sqrt{2}}{8}\right) a b+\left(\frac{2 \sqrt{6}}{3}-\sqrt{2}\right) a \\
& +\left(\frac{2 \sqrt{6}}{3}-\sqrt{2}\right) b \\
\operatorname{GA}\left(\mathscr{G}_{2}(a, b)\right)= & \left(8 \sqrt{2}+\frac{24 \sqrt{6}}{5}+12\right) a b \\
& +\left(\sqrt{3}-\frac{4 \sqrt{2}}{3}-\frac{4 \sqrt{6}}{5}+2\right) a \\
& +\left(\sqrt{3}-\frac{4 \sqrt{2}}{3}-\frac{4 \sqrt{6}}{5}+2\right) b .
\end{aligned}
$$

Proof. By using the values of the edge partition given in Table 3 in the definition of the ABC index, this index can be determined as follows:

$$
\begin{aligned}
\operatorname{ABC}\left(\mathscr{G}_{2}(a, b)\right)= & (2 a+2 b)\left(\frac{\sqrt{6}}{3}\right)+(2 a+2 b)\left(\frac{\sqrt{2}}{2}\right) \\
& +(12 a b-2 a-2 b)\left(\frac{\sqrt{2}}{2}\right) \\
& +(12 a b-2 a-2 b)\left(\frac{\sqrt{2}}{2}\right) \\
& +(12 a b)\left(\frac{2}{3}\right)=\left(\frac{12 \sqrt{2}}{8}\right) a b \\
& +\left(\frac{2 \sqrt{6}}{3}-\sqrt{2}\right) a+\left(\frac{2 \sqrt{6}}{3}-\sqrt{2}\right) b .
\end{aligned}
$$

Similarly, the GA index can be calculated as 
TABle 3: The partition of edge set of graph $\mathscr{G}_{2}(a, b)$ based on degrees of end vertices of each edge.

\begin{tabular}{lc}
\hline$\left(d_{x}, d_{y}\right)$ where $x y \in E\left(\mathscr{G}_{2}(a, b)\right)$ & Number of edges \\
\hline$(1,3)$ & $2 a+2 b$ \\
$(2,2)$ & $2 a+2 b$ \\
$(2,3)$ & $12 a b-2 a-2 b$ \\
$(2,4)$ & $12 a b-2 a-2 b$ \\
$(3,3)$ & $12 a b$ \\
\hline
\end{tabular}

TABLE 4: The partition of edge set of graph $\mathscr{G}_{2}(a, b)$ based on sum of degrees of neighbor vertices of end vertices of each edge.

\begin{tabular}{lc}
\hline$\left(S_{x}, S_{y}\right)$ where $x y \in E\left(\mathscr{G}_{2}(a, b)\right)$ & Number of edges \\
\hline$(3,7)$ & $2 a+2 b$ \\
$(4,5)$ & $2 a+2 b$ \\
$(5,7)$ & $2 a+2 b$ \\
$(7,7)$ & $a+b$ \\
$(7,8)$ & $24 a b-6 a-6 b$ \\
$(8,8)$ & $12 a b-3 a-3 b$ \\
\hline
\end{tabular}

$$
\begin{aligned}
\mathrm{GA}\left(\mathscr{G}_{2}(a, b)\right)= & (2 a+2 b)\left(\frac{\sqrt{3}}{2}\right)+(2 a+2 b)(1) \\
& +(12 a b-2 a-2 b)\left(\frac{2 \sqrt{6}}{5}\right) \\
& +(12 a b-2 a-2 b)\left(\frac{2 \sqrt{2}}{3}\right) \\
& +(12 a b)(1)=\left(8 \sqrt{2}+\frac{24 \sqrt{6}}{5}+12\right) a b \\
& +\left(\sqrt{3}-\frac{4 \sqrt{2}}{3}-\frac{4 \sqrt{6}}{5}+2\right) a \\
& +\left(\sqrt{3}-\frac{4 \sqrt{2}}{3}-\frac{4 \sqrt{6}}{5}+2\right) b .
\end{aligned}
$$

Finally, the expression of $\mathrm{ABC}_{4}$ and $\mathrm{GA}_{5}$ indices are calculated in the next theorem.
Theorem 6. The $A B C_{4}$ and $G A_{5}$ indices of the graph $\mathscr{G}_{2}(a, b)$ are

$$
\begin{aligned}
\operatorname{ABC}_{4}\left(\mathscr{G}_{2}(a, b)\right)= & \left(\frac{3 \sqrt{14}}{3}+\frac{6 \sqrt{182}}{7}\right) a b \\
& +\left(\frac{\sqrt{35}}{5}-\frac{5 \sqrt{14}}{56}+\frac{4 \sqrt{42}}{21}-\frac{3 \sqrt{182}}{14}+\frac{2 \sqrt{3}}{7}\right) a \\
& +\left(\frac{3 \sqrt{14}}{3}+\frac{6 \sqrt{182}}{7}\right) a b \\
& +\left(\frac{\sqrt{35}}{5}-\frac{5 \sqrt{14}}{56}+\frac{4 \sqrt{42}}{21}-\frac{3 \sqrt{182}}{14}+\frac{2 \sqrt{3}}{7}\right) b, \\
\mathrm{GA}_{5}\left(\mathscr{G}_{2}(a, b)\right)= & \left.\frac{32 \sqrt{14}}{5}+12\right) a b \\
& +\left(\frac{8 \sqrt{5}}{9}-\frac{8 \sqrt{14}}{5}+\frac{2 \sqrt{21}}{5}+\frac{\sqrt{35}}{3}-2\right) a \\
& +\left(\frac{8 \sqrt{5}}{9}-\frac{8 \sqrt{14}}{5}+\frac{2 \sqrt{21}}{5}+\frac{\sqrt{35}}{3}-2\right) b .
\end{aligned}
$$

Proof. To compute the values of $\mathrm{ABC}_{4}$ and $\mathrm{GA}_{5}$ indices, we need to find the partition the edge set based on the sum of degrees of the neighbors of the end vertices of each edge.
This partition is presented in Table 4. Now, using the values in the definition of $\mathrm{ABC}_{4}$ index, this index can be calculated as 


$$
\begin{aligned}
\operatorname{ABC}_{4}\left(\mathscr{G}_{2}(a, b)\right)= & (2 a+2 b)\left(\frac{2 \sqrt{42}}{21}\right)+(2 a+2 b)\left(\frac{\sqrt{35}}{10}\right)+(2 a+2 b)\left(\frac{\sqrt{14}}{7}\right)+(a+b)\left(\frac{2 \sqrt{3}}{7}\right) \\
& +(24 a b-6 a-6 b)\left(\frac{\sqrt{182}}{28}\right)+(12 a b-3 a-3 b)\left(\frac{14}{8}\right) \\
= & \left(\frac{3 \sqrt{14}}{3}+\frac{6 \sqrt{182}}{7}\right) a b+\left(\frac{\sqrt{35}}{5}-\frac{5 \sqrt{14}}{56}+\frac{4 \sqrt{42}}{21}-\frac{3 \sqrt{182}}{14}+\frac{2 \sqrt{3}}{7}\right) a+\left(\frac{3 \sqrt{14}}{3}+\frac{6 \sqrt{182}}{7}\right) a b \\
& +\left(\frac{\sqrt{35}}{5}-\frac{5 \sqrt{14}}{56}+\frac{4 \sqrt{42}}{21}-\frac{3 \sqrt{182}}{14}+\frac{2 \sqrt{3}}{7}\right) b .
\end{aligned}
$$

Similarly, the value of $\mathrm{GA}_{5}$ index can be calculated as

$$
\begin{aligned}
\mathrm{GA}_{5}\left(\mathscr{G}_{2}(a, b)\right)= & (2 a+2 b)\left(\frac{\sqrt{21}}{5}\right)+(2 a+2 b)\left(\frac{4 \sqrt{5}}{9}\right)+(2 a+2 b)\left(\frac{\sqrt{35}}{6}\right)+(a+b)(1)+(24 a b-6 a-6 b)\left(\frac{4 \sqrt{14}}{15}\right) \\
& +(12 a b-3 a-3 b)(1) \\
= & \left(\frac{32 \sqrt{14}}{5}+12\right) a b+\left(\frac{8 \sqrt{5}}{9}-\frac{8 \sqrt{14}}{5}+\frac{2 \sqrt{21}}{5}+\frac{\sqrt{35}}{3}-2\right) a\left(\frac{8 \sqrt{5}}{9}-\frac{8 \sqrt{14}}{5}+\frac{2 \sqrt{21}}{5}+\frac{\sqrt{35}}{3}-2\right) b
\end{aligned}
$$

\section{Conclusion}

The importance of computing the molecular descriptors can be understood by taking into account the recent advances in drug discovery technologies. Combinatorial chemistry, pharmacogenomics, and high-throughput screening enable us to obtain and evaluate thousands of compounds in a short period of time. These technologies pose new challenges for computational scientists as they require new approaches to computer-aided lead discovery and optimization in an accelerated way. The traditional quantitative structure-activity relationships (QSARs) are not only used to improve the biological activity of leads but also to improve their physicochemical, pharmacokinetic, and toxicological properties. Hence, the computed topological indices can be used in QSPR/QSAR studies to improve the physical/chemical properties of the considered MOFs.

\section{Data Availability}

No data were used to support this study.

\section{Conflicts of Interest}

The authors declare that there are no conflicts of interest regarding the publication of this paper.

\section{References}

[1] Y. Kinoshita, I. Matsubara, T. Higuchi, and Y. Saito, "The crystal structure of bis(adiponitrilo)copper(I) nitrate,"
Bulletin of the Chemical Society of Japa, vol. 32, pp. 1221-1226, 1959.

[2] B. F. Hoskins and R. Robson, "Infinite polymeric frameworks consisting of three dimensionally linked rod-like segments," Journal of the American Chemical Society, vol. 111, pp. 5962-5964, 1989.

[3] J. Lee, O. K. Farha, J. Roberts, K. A. Scheidt, S. T. Nguyen, and J. T. Hupp, "Metal-organic framework materials as catalysts," Chemical Society Reviews, vol. 38, no. 5, pp. 1450-1459, 2009.

[4] E. A. Hall, L. R. Redfern, M. H. Wang, and K. A. Scheidt, "Lewis acid activation of a hydrogen bond donor metal-organic framework for catalysis," ACS Catalysis, vol. 6, no. 5, pp. 3248-3252, 2016.

[5] S. Roy, C. B. George, and M. A. Ratner, "Catalysis by a zincporphyrin-based metal-organic framework: from theory to computational design," The Journal of Physical Chemistry C, vol. 116, no. 44, pp. 23494-23502, 2012.

[6] P. Horcajada, C. Serre, G. Maurin et al., "Flexible porous metal-organic frameworks for a controlled drug delivery," Journal of the American Chemical Society, vol. 130, no. 21, pp. 6774-6780, 2008.

[7] M. Vallet-Reg, F. Balas, and D. Arcos, "Mesoporous materials for drug delivery," Angewandte Chemie International Edition, vol. 46, pp. 7548-7558, 2007.

[8] B. Mandal, J. S. Chung, and S. G. Kang, "Exploring the geometric, magnetic and electronic properties of Hofmann MOFs for drug delivery," Physical Chemistry Chemical Physics, vol. 19, no. 46, pp. 31316-31324, 2017.

[9] L. Sarkisov, "Toward rational design of metal-organic frameworks for sensing applications: efficient calculation of adsorption characteristics in zero loading regime," The Journal of Physical Chemistry C, vol. 116, no. 4, pp. 3025-3033, 2012.

[10] C. Chakravarty, B. Mandal, and P. Sarkar, "Bis(dithioline) based metal-organic frameworks with superior electronic and 
magnetic properties: spin frustration to spintronics and gas sensing," The Journal of Physical Chemistry C, vol. 120, no. 49, pp. 28307-28319, 2016.

[11] J.-R. Li, R. J. Kuppler, and H.-C. Zhou, "Selective gas adsorption and separation in metal-organic frameworks," Chemical Society Reviews, vol. 38, no. 5, pp. 1477-1504, 2009.

[12] J. Y. Kim, R. Balderas-Xicohténcatl, L. Zhang et al., "Exploiting diffusion barrier and chemical affinity of metalorganic frameworks for efficient hydrogen isotope separation," Journal of the American Chemical Society, vol. 139, no. 42, pp. 15135-15141, 2017.

[13] L. J. Murray, M. Dincă, and J. R. Long, "Hydrogen storage in metal-organic frameworks," Chemical Society Reviews, vol. 38, no. 5, pp. 1294-1314, 2009.

[14] N. L. Rosi, J. Eckert, M. Eddaoudi et al., "Hydrogen storage in microporous metal-organic frameworks," Science, vol. 300, no. 5622, pp. 1127-1129, 2003.

[15] R. D. Kennedy, V. Krungleviciute, D. J. Clingerman et al., "Carborane-based metal-organic framework with high methane and hydrogen storage capacities," Chemistry of Materials, vol. 25, no. 17, pp. 3539-3543, 2013.

[16] A. U. Czaja, N. Trukhan, and U. Müller, "Industrial applications of metal-organic frameworks," Chemical Society Reviews, vol. 38, no. 5, pp. 1284-1293, 2009.

[17] S. J. Geier, J. A. Mason, E. D. Bloch et al., "Selective adsorption of ethylene over ethane and propylene over propane in the metal-organic frameworks $\mathrm{M}_{2}$ (dobdc) $(\mathrm{M}=\mathrm{Mg}, \mathrm{Mn}, \mathrm{Fe}, \mathrm{Co}$, Ni, Zn)," Chemical Science, vol. 4, no. 5, pp. 2054-2061, 2013.

[18] B. Mu, P. M. Schoenecker, and K. S. Walton, "Gas adsorption study on mesoporous Metal-Organic framework UMCM-1," The Journal of Physical Chemistry C, vol. 114, no. 14, pp. 6464-6471, 2010.

[19] W. L. Queen, M. R. Hudson, E. D. Bloch et al., "Comprehensive study of carbon dioxide adsorption in the metalorganic frameworks $\mathrm{M}_{2}$ (dobdc) $(\mathrm{M}=\mathrm{Mg}, \mathrm{Mn}, \mathrm{Fe}, \mathrm{Co}, \mathrm{Ni}, \mathrm{Cu}$, Zn)," Chemical Science, vol. 5, no. 12, pp. 4569-4581, 2014.

[20] J. Park, J. D. Howe, and D. S. Sholl, "How reproducible are isotherm measurements in metal-organic frameworks?" Chemistry of Materials, vol. 29, no. 24, Article ID 10487, 2017.

[21] H. Wu, W. Zhou, and T. Yildirim, "High-capacity methane storage in Metal-Organic frameworks $\mathrm{M}_{2}(\mathrm{dhtp})$ : the important role of open metal sites," Journal of the American Chemical Society, vol. 131, no. 13, pp. 4995-5000, 2009.

[22] P. D. C. Dietzel, V. Besikiotis, and R. Blom, "Application of metal-organic frameworks with coordinatively unsaturated metal sites in storage and separation of methane and carbon dioxide," Journal of Materials Chemistry, vol. 19, no. 39, pp. 7362-7370, 2009.

[23] K. Lee, J. D. Howe, L.-C. Lin, B. Smit, and J. B. Neaton, "Smallmolecule adsorption in open-site metal-organic frameworks: a systematic density functional theory study for rational design," Chemistry of Materials, vol. 27, no. 3, pp. 668-678, 2015.

[24] H. Rodhe, "A comparison of the contribution of various gases to the greenhouse effect," Science, vol. 248, no. 4960, p. 1217, 1990.

[25] A. Singh and M. Agrawal, "Acid rain and its ecological consequences," Journal of Environmental Biology, vol. 29, p. 15, 2007.

[26] J. S. Olson and G. N. Phillips Jr., "Myoglobin discriminates between $\mathrm{O}_{2}, \mathrm{NO}$, and $\mathrm{CO}$ by electrostatic interactions with the bound ligand," JBIC Journal of Biological Inorganic Chemistry, vol. 2, no. 4, pp. 544-552, 1997.

[27] J. D. Howe, Y. Liu, L. Flores, D. A. Dixon, and D. S. Sholl, "Acid gas adsorption on metal-organic framework nanosheets as a model of an "All-Surface" material," Journal of Chemical Theory and Computation, vol. 13, no. 3, pp. 1341-1350, 2017.

[28] K. Tan, S. Zuluaga, H. Wang et al., "Interaction of acid gases $\mathrm{SO}_{2}$ and $\mathrm{NO}_{2}$ with coordinatively unsaturated metal organic frameworks: M-MOF-74 (M= Zn, Mg, Ni, Co)," Chemistry of Materials, vol. 29, no. 10, pp. 4227-4235, 2017.

[29] K. Yu, K. Kiesling, and J. R. Schmidt, "Trace flue gas contaminants poison coordinatively unsaturated metal-organic frameworks: implications for $\mathrm{CO}_{2}$ adsorption and separation," The Journal of Physical Chemistry C, vol. 116, no. 38, pp. 20480-20488, 2012.

[30] J. A. Bondy and U. S. Murty, Graph Theory and its Application, The Macmillan Press, London, UK, 1976.

[31] H. van de Waterbeemd, R. E. Carter, G. Grassy et al., "Glossary of terms used in computational drug design (IUPAC recommendation 1997)," Annual Reports in Medicinal Chemistry, vol. 33, pp. 397-409, 1998.

[32] A. R. Katritzky and E. V. Gordeeva, "Traditional topological indices vs. electronic, geometrical, and combined molecular descriptors in QSAR/QSPR research," Journal of Chemical Information and Computer Sciences, vol. 33, pp. 835-857, 1993.

[33] A. T. Balaban, "Topological and stereochemical molecular descriptors for databases useful in QSAR, similarity/dissimilarity and drug design," SAR and QSAR in Environmental Research, vol. 8, p. 121, 1998.

[34] J. Devillers, "New trends in (Q)SAR modeling with topological indices," Current Opinion in Drug Discovery \& Development, vol. 3, pp. 275-279, 2000.

[35] H. Wiener, "Structural determination of paraffin boiling points," Journal of the American Chemical Society, vol. 69, no. 1, pp. 17-20, 1947.

[36] M. Randic, "Characterization of molecular branching," Journal of the American Chemical Society, vol. 97, no. 23, pp. 6609-6615, 1975.

[37] B. Bollobás and P. Erdos, "Graphs of extremal weights," Ars Combinatoria, vol. 50, pp. 225-233, 1998.

[38] I. Gutman and N. Trinajsti, "Graph theory and molecular orbitals. III. Total $\varphi$-electron energy of alternant hydrocarbon," Chemical Physics Letters, vol. 17, pp. 535-538, 1972.

[39] G. H. Shirdel, H. R. Pour, and A. M. Sayadi, "The hyperZagreb index of graph operations, Iran," The Journal of Mathematical Chemistry, vol. 4, pp. 213-220, 2013.

[40] S. H. Bertz and W. F. Wright, Graph Theory Notes of New York, Vol. 35, New York Academy of Sciences, New York, NY, USA, 1998.

[41] S. Nikoli, I. M. Toli, and N. Trinajsti, "On the complexity of molecular graphs," MATCH Communications in Mathematical and in Computer Chemistry, vol. 40, pp. 187-201, 1999.

[42] S. Nikoli, N. Trinajsti, and I. M. Toli, "Walk counts, labyrinthicity, and complexity of acyclic and cyclic graphs and molecules," Journal of Chemical Information and Computer Sciences, vol. 40, no. 1, pp. 920-926, 2000.

[43] S. Nikoli, I. M. Toli, N. Trinajsti, and I. Bau i, "On the Zagreb indices as complexity indices," Croatica Chemica Acta, vol. 73, pp. 909-921, 2000.

[44] S. Nikoli, N. Trinajsti, I. M. Toli, G. Rcker, and C. Rcker, Complexity in Chemistry, D. Bonchev and D. H. Rouvray, Eds., .

[45] M. Ghorbani and M. A. Hosseinzadeh, "Computing ABC4 index of nanostar dendrimers," Optoelectronics and Advanced Materials: Rapid Communications, vol. 4, no. 9, pp. 14191422, 2010. 
[46] A. Golbraikh, D. Bonchev, and A. Tropsha, "Novel ZEisomerism descriptors derived from molecular topology and their application to QSAR analysis," Journal of Chemical Information and Computer Sciences, vol. 42, no. 39, pp. 769-787, 2002.

[47] A. Milicevic and S. Nikolic, "On variable zagreb indices," Croatica Chemica Acta, vol. 77, no. 1-2, pp. 97-101. in press, 2004.

[48] A. T. Balaban, "Chemical graphs," Theoretica Chimica Acta, vol. 53, no. 4, pp. 355-375, 1979.

[49] D. Bonchev and N. Trinajsti, "Overall molecular descriptors. 3. Overall Zagreb indices," SAR and QSAR in Environmental Research, vol. 12, no. 1-2, pp. 213-236, 2001.

[50] J. Devillers and A. T. Balaban, Topological Indices and Related Descriptors in QSAR and QSPR, Gordon \& Breach, Amsterdam, Netherlands, 1999.

[51] A. Golbraikh, D. Bonchev, and A. Tropsha, "Novel chirality descriptors derived form molecular topology," Journal of Chemical Information and Computer Sciences, vol. 41, no. 1, pp. 147-158, 2001.

[52] M. Karelson, Molecular Descriptors in QSAR/QSPR, WileyInterscience, New York, NY, USA, 2000.

[53] R. Todeschini and V. Consonni, Handbook of Molecular Descriptors, Wiley VCH, Weinheim, Germany, 2000.

[54] B. Zhou and N. Trinajstic, "On a novel connectivity index," The Journal of Mathematical Chemistry, vol. 46, pp. 12521270, 2009.

[55] B. Zhou and N. Trinajstic, "On general sum-connectivity index," The Journal of Mathematical Chemistry, vol. 47, pp. 210-218, 2010.

[56] E. Estrada, L. Torres, L. Rodriguez, and I. Gutman, “An atombond connectivity index: modelling the enthalpy of formation of alkanes," Indian Journal of Chemistry, vol. 37A, pp. 849855, 1998.

[57] E. Estrada, "Generalization of topological índices," Chemical Physics Letters, vol. 336, no. 3-4, pp. 248-252, 2001.

[58] B. Vukičević and B. Furtula, "Topological index based on the ratios of geometrical and arithmetical means of end-vertex degrees of edges," Journal of Mathematical Chemistry, vol. 46, no. 4, pp. 1369-1376, 2009.

[59] A. Graovac, M. Ghorbani, and M. A. Hosseinzadeh, "Computing fifth geometric-arithmetic index for nanostar dendrimers," Journal of Mathematical Nanoscience, vol. 1, no. 1-2, pp. 32-42, 2011.

[60] W. Gao, M. Aamir, Z. Iqbal, M. Ishaq, and A. Aslam, "On irregularity measures of some dendrimers structures," Mathematics, vol. 7, no. 3, p. 271, 2019.

[61] Z. Iqbal, M. Ishaq, A. Aslam, and W. Gao, "On eccentricitybased topological descriptors of water-soluble dendrimers," Zeitschrift Fr Naturforschung C, vol. 74, no. 1-2, pp. 25-33, 2019.

[62] A. Aslam, J. L. G. Guirao, S. Ahmad, and W. Gao, "Topological indices of line graph of subdivision graph of complete bipartite graphs," Applied Mathematics and Information Sciences, vol. 11, no. 6, pp. 1631-1636, 2017.

[63] W. Gao, Z. Iqbal, M. Ishaq, R. Sarfraz, M. Aamir, and A. Aslam, "On eccentricity-based topological indices study of a class of porphyrin-cored dendrimers," Biomolecules, vol. 8, no. 3, p. 71, 2018.

[64] Z. Iqbal, A. Aslam, M. Ishaq, and M. Aamir, "Characteristic study of irregularity measures of some nanotubes," Canadian Journal of Physics, vol. 97, no. 10, pp. 1125-1132, 2019.
[65] W. Gao, S. Akhter, Z. Iqbal, M. Qasim, and A. Aslam, "The topological aspects of phthalocyanines and porphyrins dendrimers," IEEE Access, vol. 8, pp. 168631-168649, 2020.

[66] B. Wurster, D. Grumelli, D. Hötger, R. Gutzler, and K. Kern, "Driving the oxygen evolution reaction by nonlinear cooperativity in bimetallic coordination catalysts," Journal of the American Chemical Society, vol. 138, no. 11, pp. 3623-3626, 2016.

[67] A. J. Clough, J. W. Yoo, M. H. Mecklenburg, and S. C. Marinescu, "Two-dimensional metal organic surfaces for efficient hydrogen evolution from water," Journal of the American Chemical Society, vol. 137, pp. 118-121, 2014. 